\title{
Women's experiences receiving abnormal prenatal chromosomal microarray testing results
}

\author{
Barbara A. Bernhardt, $\mathrm{MS}^{1,2}$, Danielle Soucier, $\mathrm{MBE}^{2}$, Karen Hanson, $\mathrm{MS}^{3}$, Melissa S. Savage, $\mathrm{MS}^{3}$, \\ Laird Jackson, MD ${ }^{4}$ and Ronald J. Wapner, $\mathrm{MD}^{3}$
}

Purpose: Genomic microarrays can detect copy-number variants not detectable by conventional cytogenetics. This technology is diffusing rapidly into prenatal settings even though the clinical implications of many copy-number variants are currently unknown. We conducted a qualitative pilot study to explore the experiences of women receiving abnormal results from prenatal microarray testing performed in a research setting.

Methods: Participants were a subset of women participating in a multicenter prospective study "Prenatal Cytogenetic Diagnosis by Array-based Copy Number Analysis." Telephone interviews were conducted with 23 women receiving abnormal prenatal microarray results.
Results: We found that five key elements dominated the experiences of women who had received abnormal prenatal microarray results: an offer too good to pass up, blindsided by the results, uncertainty and unquantifiable risks, need for support, and toxic knowledge.

Conclusion: As prenatal microarray testing is increasingly used, uncertain findings will be common, resulting in greater need for careful pre- and posttest counseling, and more education of and resources for providers so they can adequately support the women who are undergoing testing.

Genet Med 2013:15(2):139-145

Key Words: genetic counseling; microarray testing; prenatal diagnosis; qualitative research

\section{INTRODUCTION}

Chromosomal microarrays (CMAs) can detect genetic copynumber variants (CNVs) not detectable by conventional cytogenetics. In children with unexplained developmental delay, autism spectrum disorder, or multiple congenital anomalies, CMA testing explains the child's disorder in nearly $20 \%$ of cases. ${ }^{1}$ Although the American College of Obstetricians and Gynecologists recommends that CMAs be offered prenatally only when a fetal anomaly is present and karyotyping is normal, ${ }^{2}$ it is being used increasingly for other indications, including advanced maternal age, increased risk after maternal serum marker screening, and maternal anxiety. ${ }^{3-5}$

Although most prenatal CMA testing will yield normal results, a CNV of known or probable clinical significance not detectable by karyotypic analysis is found in between 1.6 and $6.0 \%$ of cases, depending on reason for referral. ${ }^{6}$ The impact of positive CMA results on women is likely to be substantial because any indication of a problem with the fetus can transform the experience of pregnancy. ${ }^{7,8}$ Prenatal microarray testing is generally offered in conjunction with pretest genetic counseling to review possible test outcomes and the potential risks, benefits, and limitations of testing. ${ }^{9}$ The extent to which this counseling prepares couples for abnormal test results is currently unclear. For those couples receiving positive results, counseling and decision-making is complicated because the expected phenotype associated with most prenatally detected CNVs is either unknown or based on children with an observed clinical abnormality and therefore biased toward the more severe end of the phenotypic spectrum..$^{10,11}$

Studies of genetic counseling issues associated with prenatal microarray testing and patients' emotional response to testing have been recommended., ${ }^{9,11}$ Accordingly, we conducted a small pilot study to explore the experiences of women who received positive results from prenatal microarray testing.

\section{Recruitment}

\section{MATERIALS AND METHODS}

Participants in this study were a subset of women participating in a multicenter prospective study "Prenatal Cytogenetic Diagnosis by Array-based Copy Number Analysis," coordinated through Columbia University. ${ }^{6}$ The study enrolled 4,450 women who received genetic counseling and were undergoing an invasive prenatal diagnostic procedure for karyotype analysis for standard indications. During an informed consent session taking place during or immediately after the genetic counseling session, women were informed about the prenatal microarray study and given information about the risks, benefits, and limitations of CMA testing. The consent sessions were conducted either by a genetic counselor or a study coordinator trained by the Columbia team. Blood samples were collected from both 
parents at the time of consent. CMA testing was performed in one of four study laboratories, and standard karyotype analysis was performed in a fifth laboratory. The arrays were designed to detect more than 80 known deletion/duplication syndromes, and any CNV larger than $1 \mathrm{MB}$ in the backbone. Participating women received results from cytogenetic analysis 7-10 days after specimen collection, and microarray results were reported 1-2 weeks later. Any result of uncertain clinical significance was reviewed along with clinical information by a clinical advisory committee composed of genetic specialists, physicians, and genetic counselors. The committee decided if the finding was potentially clinically relevant and hence reported to the patient. $\mathrm{CNV}$ results classified as likely benign were not returned to the patient. Most results were returned to participants by a study genetic counselor and the rest by a study obstetrician, and both were available for follow-up counseling.

Eligible participants for this study were recruited from participants enrolled during the last three years of the five-year parent study who agreed to be contacted for follow-up, were English speaking, were 6 months postpartum or post-pregnancy termination, and had received either positive or uncertain CMA results. The principal investigator of the parent study sent a letter to eligible women describing the study. Interested participants contacted the University of Pennsylvania research coordinator to provide consent and set up a telephone interview. This study was approved by the institutional review board of the University of Pennsylvania.

\section{Data collection}

Interviews were conducted using an interview guide to elicit the women's perspectives on their experience with CMA testing by addressing reasons for pursuing prenatal testing and participating in the microarray study; understanding of microarray testing; receiving results; decision making after receiving results; emotional response to results; and needs during the process of microarray testing and afterward. The questions were openended and probes were used to elaborate on responses. The interviews lasted between 45 and $60 \mathrm{~min}$ and were recorded, transcribed verbatim, and de-identified.

\section{Data analysis}

All transcripts were uploaded to the qualitative analysis software NVivo 8 for coding and analysis. A codebook was developed and revised based on themes as they emerged from the data. Two investigators coded the transcripts after reaching standard intercoder reliability. In-depth analysis of the coded data was conducted using a grounded theory approach to document and interpret themes and to explore similarities and differences between participants' experiences.

\section{RESULTS}

Fifty-eight women were eligible and invited to participate. Twentyfour women responded indicating interest in being interviewed, and 23 women were interviewed. The indications for prenatal testing, type of test, and results are included in Table 1.
Table 1 Reasons for testing and results among 23 participants

\begin{tabular}{|c|c|}
\hline & $\begin{array}{l}\text { Number of } \\
\text { participants }\end{array}$ \\
\hline \multicolumn{2}{|l|}{ Type of procedure } \\
\hline Amniocentesis & 13 \\
\hline Chorionic villus sampling & 10 \\
\hline \multicolumn{2}{|l|}{ Indication for procedure } \\
\hline Abnormal ultrasound & 7 \\
\hline $\begin{array}{l}\text { Routine care (advanced maternal age, positive } \\
\text { screening, etc.) }\end{array}$ & 16 \\
\hline \multicolumn{2}{|l|}{ Result } \\
\hline $\begin{array}{l}\text { Inherited deletion/duplication—-known } \\
\text { pathogenic }\end{array}$ & 2 \\
\hline $\begin{array}{l}\text { Inherited deletion/duplication-potentially } \\
\text { clinically significant }\end{array}$ & 10 \\
\hline $\begin{array}{l}\text { De novo deletion/duplication—known } \\
\text { pathogenic }\end{array}$ & 7 \\
\hline $\begin{array}{l}\text { De novo deletion/duplication_-potentially } \\
\text { clinically significant }\end{array}$ & 4 \\
\hline \multicolumn{2}{|l|}{ Outcome } \\
\hline Continued pregnancy & 16 \\
\hline Terminated & 7 \\
\hline
\end{tabular}

The following key themes were identified through analysis, and quotes to illustrate each theme are included.

\section{An offer too good to pass up}

Because they had already decided to have an invasive procedure for fetal karyotype analysis, all women indicated that they saw no reason to decline microarray testing. Thus, the decision regarding microarray testing was easy to make, because the women could get more information about their baby's health at no additional cost or risk. One woman said:

"I just heard like "oh my gosh, you can have these 80 other tests" and I thought, well, 80 is better than what I'm getting, so ..." (Participant 9)

Because it seemed risk-free, many women said they had not understood much about microarray testing before having it done. This was especially true of the women carrying a fetus with a structural anomaly, most of whom admitted to being distressed during the informed consent session. When asked what she understood when she consented to the study, one woman who had just been informed of her baby's ultrasound anomalies said:

"At that time, probably not a lot just because we were under emotional distress .... She [the genetic counselor] was talking and we were just crying. (Participant 3)

\section{Blindsided by the results}

Because of the research protocol design, women received their karyotype results before the array results. When 
receiving abnormal CMA results after first receiving normal cytogenetic results, many women reported being blindsided. One woman said:

"Once we got the results of the chorionic villus sampling I actually thought we were in the clear and we had started telling people that I was pregnant .... I was totally shocked when they called and said there was an issue [on the microarray]." (Participant 13)

\section{Another woman recalled:}

"I remember getting three phone calls from the genetic counselor. One was saying preliminary results are in and everything's fine. Second one was we got the next seteverything's fine. The third call was "hey, can you call me back." And then I kind of panicked.” (Participant 5)

Women who had CMA testing because of a fetal ultrasound anomaly generally were not blindsided by results. These women anticipated abnormal results, having already been told that something was wrong with their baby.

\section{Uncertainty and unquantifiable risks}

For most of the women, the abnormal microarray results left them initially shocked, anxious, confused, and overwhelmed. As they attempted to sort out the meaning of the CMA results, they were often left with lingering uncertainties. One woman said:

"You know, they're telling me there's something wrong, but they can't tell me what.... We wanted to know what that would mean for our son in the future. And they really couldn't tell us." (Participant 18)

Some women receiving uncertain microarray results believed that if they searched hard enough, they could ultimately find definitive information that could aid in making an informed decision about their pregnancy. Some women were confused about whether their baby's problem was serious because they received conflicting information from counselors and physicians, and found additional contradictory information on the Internet. One woman, who was told at 24 weeks that her fetus had a de novo deletion, described her search for additional information:

"I started getting really panicky that the child that I was carrying was going to be severely autistic with seizures and schizophrenia.... I would look online and I met with a geneticist and talked to an autism specialist. And frankly nobody could really tell me.... I ended up going to a crisis counselor because it was very stressful." (Participant 7)

For some women, even when a well-described disorder such as the 22q11.2 deletion syndrome was diagnosed, making a decision about the pregnancy was difficult because of the wide phenotype spectrum. One woman who terminated a pregnancy diagnosed with a de novo DiGeorge deletion said:

"We still grapple with this because it is very much a spec-
trum of severity, very, very hard to predict what the outcome
would be.... So that was very, very difficult for us because it
made assessing our choices really hard." (Participant 20)

Many women discussed the difficulty of emotionally and intellectually managing the uncertainties of results given how far along they were in their pregnancies. They wanted to make decisions based on complete understanding of the microarray findings but didn't have enough time to gather and understand the information, especially immediately after receiving shocking news. One woman explained:

"I think what is so difficult about these decisions-on top of the shock of it, the helplessness-is the timing.... We felt like we were desperately trying to build enough information to make an informed decision in a very fast amount of time and that was very, very stressful for us." (Participant 16)

Several women who had testing because of a fetal structural anomaly were not as concerned about the uncertainties because any positive result provided confirmation that their baby really did have a problem and justified pregnancy termination. One woman said:

"Well the baby was diagnosed with tetralogy of Fallot. She [the genetic counselor] said: "the testing revealed that the baby does have a $22 \mathrm{q}$ deletion." So the writing was on the wall at that point." (Participant 11)

\section{Need for support}

These women needed support to manage, understand, and act on the microarray results. Most women did receive help in the form of emotional support, information, answers to questions, and referrals from a health-care provider, often an obstetrician or a study genetic counselor. One woman said:

"The genetic counselor probably called me two or three times after our initial conversation about the results to see how we were doing and if there was anything else she could do for us.... She did a very nice job making herself and different resources available." (Participant 10)

Many of the women commented that they did not get as much support as they wanted with decision-making about continuing or terminating their pregnancy. Several women sought advice from genetic counselors about what to do but were only told again that information on the anticipated outcome of the pregnancy was not available. Three of the women mentioned that a senior geneticist helped them because his experience and reassuring manner led them to end their personal frenzied search for additional information. Several women also mentioned that 


\section{ORIGINAL RESEARCH ARTICLE}

speaking with an expert about their child's disorder provided the information they needed to make a decision about the pregnancy.

The majority of participants received support from their partners and family members. This support was especially important immediately after receiving microarray results when the distraught women were trying to understand and absorb the implications of the findings. One woman said:

"I just wasn't grasping or paying attention ... but thank goodness my husband-he was like absorbing everything and he just really understood ... intellectually I guess I was prepared but everything else was not prepared." (Participant 15)

Several of the women continuing their pregnancies reported that providers were not supportive of their decision. One woman continuing her pregnancy with a 22q11.2 deletion reported being asked multiple times if she was terminating. She said:

"Abortion ... they asked us like four different times, so they're like "are you sure, are you sure?" It got to the point where we were like "just don't ask us because we're getting upset about this."” (Participant 19)

\section{Toxic knowledge}

Most women understood that CMA testing could identify serious problems in the baby missed on routine cytogenetic analysis. Only one woman recalled having been told during the informed consent session that some results might be uninterpretable or uncertain. When they were given such results, many women considered this information to be knowledge they wish they did not have ("toxic knowledge"). Instead of being excited about the pregnancy, the women were anxious and constantly wondering if the CNV would affect the baby's health. One woman said:

\section{"To know that your child may have that takes away some of the glow of being pregnant, because now you're thinking when you have this child, what is that gonna look like, how is that gonna feel, how do I cope with that? ... now you have an added element you have to deal with." (Participant 4)}

Another woman said:

"It took us two or three more months after the tests to even buy the crib and do the nursery stuff ... because we didn't know what was gonna happen ... then after that our OB was always checking to see if he was still alive in the womb ... it just kind of put us off from enjoying our pregnancy." (Participant 15)

Even after delivering a normal-appearing baby, 8 of the 16 women who continued their pregnancies admitted to lingering worries about their child's development. These women reported
BERNHARDT et al | Receiving abnormal prenatal chromosomal microarray testing results

watching the baby carefully for any health issues or developmental delays. One woman explained:

"Since I had this uncertain microarray result ... if anything happens to him in the future ... that will always pop up in my mind... You just have to have a "wait and see" attitude.... I'm a lot more vigilant." (Participant 8)

Half of the study participants or their partner carried a deletion or duplication that was passed on to their baby. Sometimes this information was helpful because the presence of the variant in a normal parent gave the couple hope that their baby could be unaffected. The finding of a parental variant often prompted providers' questions to determine if the carrier parent was phenotypically normal, leading the parent to question whether he or she was actually normal. One woman whose husband carried a deletion told about being informed:

"The first thing the genetic counselor said-and she said it very delicately - is "so does your husband have a little head?" So I was like "No, I think my husband's head is pretty normal." And she said that the only condition present in this other person who had a similar chromosomal issue was microcephaly.... Well, it really messed him up." (Participant 12)

One woman who carried the same deletion as her fetus said she was repeatedly asked if she had any abnormalities:

"Once again, he's asking me if I have a learning disability and I actually felt kind of insulted ... he kept insisting that I come in and I kind of got a feeling he was trying to make me come in because he wanted to see for himself whether I had a learning disability." (Participant 16)

\section{DISCUSSION}

This study is the first to explore women's experiences with positive prenatal microarray findings. We found that five key elements dominated the experiences of women who had testing as a part of a research protocol: an offer too good to pass up, blindsided by the results, uncertainty and unquantifiable risks, need for support, and toxic knowledge.

Over the past few decades, prenatal testing and screening have become so routinized that an invitation to learn information about the health of their unborn child is delivered with the expectation that women will accept the offer. ${ }^{14}$ By embracing the "knowledge is power" notion, many women opt for testing without the opportunity to carefully weigh the risks, benefits, and consequences of the test. ${ }^{15-17}$ This was especially prevalent among women in our study who were already having amniocentesis or chorionic villus sampling for karyotypic analysis and who were able to obtain microarray testing without additional risk or cost.

Because participants had already received news that their baby's karyotype was normal, it should not be surprising 
that many were blindsided by the abnormal microarray findings. Although some of the shock associated with receiving abnormal results was an artifact of the study design, pregnant women are rarely prepared to receive such emotionally devastating news ${ }^{8,18,19}$ Moreover, immediately after receiving results, women need to make a time-sensitive decision about pregnancy termination that is both cognitively and emotionally demanding. ${ }^{20,21}$

Due to the uncertainties associated with some CMA results, such decision making is particularly demanding. Unlike the postnatal situation in which microarray testing is ordered to explain a child's clinical phenotype, these women were not able to interpret the findings in light of their child's observable health and development. Both the phenotypic range associated with many CNVs, and the lack of precise probability of a medical issue being present challenged a woman's ability to imagine how her child might be affected. Although some uncertainty associated with prenatal microarray testing could be reduced by the use of targeted arrays, ${ }^{22}$ uncertainty will still remain because of the variability of phenotype of even well-described conditions such as the 22q11.2 microdeletion syndrome. ${ }^{23}$ In our study, among the six women whose fetuses were diagnosed with deletions involving the DiGeorge region, two chose to terminate and four continued their pregnancies. Most of these women described their decision making as tortured, and women continuing their pregnancies experienced considerable uncertainty about the development of their child after delivery.

After receiving results, women attempted to manage the uncertainty by quickly gathering information about the implication of the finding. This search proved difficult for most women, due both to their emotional turmoil and the lack of available information. As women grappled with the emotional fallout of results, they wanted their providers to both provide information and facilitate decision making. Although many women did receive adequate support, some felt abandoned by their providers as they struggled to make a decision. Consistent with other reports, ${ }^{24,25}$ some women said that their decision to continue their pregnancy was questioned by health-care providers and they needed to justify their decision to both themselves and others.

When women were informed that their baby may or may not have a problem of variable severity, some women regretted having been given such information. For many women continuing their pregnancies, the experience of pregnancy as well as the joy of watching their baby develop was changed by the microarray results. "Watchful waiting" became the norm, and concerns weren't totally alleviated by normal sonograms or by delivering a child who appeared normal at birth and during infancy. Although the longer-term impact on the parent-child relationship cannot be determined by this study, in other situations in which an abnormality has been suspected, such as a positive newborn screening test followed by normal follow-up testing, parents' concern about their child's health and development continues long after birth. ${ }^{26,27}$
Although some of the women interviewed were relieved to learn that they or their partner shared a CNV with their baby, there were some instances in which such knowledge caused distress. Similar to the experiences of parents learning such information when their child is tested after birth, some individuals felt an altered sense of identity upon learning that they carry a CNV that is present in people with pathological features. ${ }^{28}$

\section{Limitations}

This was a qualitative study with a small number of participants. We cannot determine whether the eligible women who chose to be interviewed differ in significant ways from those who did not participate. All of the women interviewed had microarray testing as a part of a research protocol, and women who participate in research may not be representative of the general population of pregnant women. We interviewed only women receiving positive results, so we cannot comment on the experiences of women receiving normal CMA results. Furthermore, qualitative studies aim to identify a broad range of experiences and our findings are not intended to quantify the prevalence of any particular experience.

\section{Clinical implications and conclusions}

Consistent with other reports, ${ }^{18-19,24}$ most of the women interviewed experienced intense negative emotional reactions when given abnormal prenatal test results. Although little can be done to mitigate the immediate acute psychological reaction to receiving such news, providers can help patients make meaning of results and move toward making well-informed decisions.

Our findings suggest that a thorough discussion of the various uncertainties associated with abnormal microarray results, as well as the couple's tolerance for uncertainty, needs to be part of pretest genetic counseling. Although the possibility of uncertain findings was included in all pretest informed consent sessions, the reality of such findings was not truly considered. Discussion of uncertain prenatal microarray findings or findings consistent with a variable phenotype is especially important because positive findings frequently include disorders that are less severe or of more variable phenotype than disorders found on karyotyping. Other research has suggested that patients who make a decision based on understanding the limitations of procedures are more likely to be prepared for abnormal results, and less likely to have adverse psychological reactions. ${ }^{29}$

Prenatal diagnosis counseling needs to focus on factors important to the women who are making decisions. ${ }^{30-32}$ Genetic counseling generally emphasizes genetic diseases, procedures, and risks. Other factors, such as anxiety associated with testing, and options and decision making if an abnormality is found, may be important to women, but not discussed during pretest genetic counseling. ${ }^{33}$ In addition, genetic counseling frequently ignores the social and moral values of patients, their views toward abortion, and their attitudes toward parenting a 
child with a disability. ${ }^{33,34} \mathrm{~A}$ discussion of these issues before undergoing prenatal testing may better prepare couples who are faced with making a decision in the midst of a crisis.

Genetic counselors and prenatal care providers will need to devote considerable time to carefully educating, counseling, and supporting women and their partners as they receive abnormal microarray results, make a decision about continuing their pregnancy, and cope with the aftermath of their decision. Previous research has documented that giving bad news, coping with uncertain information, and facilitating decision making, especially in the face of uncertainty, are among the most distressing tasks faced by genetic counselors. ${ }^{35,36}$ Unfortunately, to reduce their own distress, some genetic counselors may withdraw from clients in these situations. Support for and training of clinicians to deal with uncertainty and assist clients making decisions based on uncertain information are needed. In addition, both providers and patients must understand and acknowledge that there will be scientific uncertainty associated with many CMA results. Because of the time-sensitive decisions couples need to make, clinical geneticists and genetic counselors can be helpful by having information about the clinical consequences of the microarray finding on hand at the time of results disclosure. To facilitate this, the International Standards for Cytogenomic Arrays Consortium is developing a publically available database of the potential clinical relevance of CNVs throughout the genome. ${ }^{37}$

Given that most couples experience difficulty understanding and absorbing information immediately after receiving devastating news, genetic counselors and obstetricians may need to have multiple contacts with the couple. Couples choosing pregnancy termination should be given assistance making all arrangements for the abortion in a timely manner. Couples continuing pregnancies should be contacted periodically to assess ongoing needs, and a care plan for the child after birth should be discussed. Although many couples are able to locate others who have a child with a similar disorder, genetic counselors should help families forge connections with other families.

New technologies aimed at increasing the sensitivity and specificity of prenatal testing and reducing risk to the pregnancy are advancing rapidly. It is highly likely that in the near future, fetal microarray testing will become the first tier-test for all invasive prenatal testing. ${ }^{38,39}$ As this shift occurs, there will be a need for careful pre- and posttest counseling, and more education of providers so they can adequately support women who are undergoing testing.

\section{ACKNOWLEDGMENTS}

This work was supported by funding from the National Human Genome Research Institute, National Institutes of Health (P50HG004487) and from the National Institute of Child Health and Development, National Institutes of Health (R01HD05565101 and R01HD055651-03S1).

\section{DISCLOSURE}

The authors declare no conflict of interest.

\section{REFERENCES}

1. Manning M, Hudgins L. Array-based technology and recommendations for utilization in medical genetics practice for detection of chromosomal abnormalities. Genet Med 2010;12:742-745.

2. Committee on Genetics. ACOG Committee Opinion No. 446: Array Comparative Genomic Hybridization in Prenatal Diagnosis. Obstet Gynecol 2009;114:11611163.

3. Novelli A, Grati FR, Ballarati L, et al. Microarray application in prenatal diagnosis: a position statement from the cytogenetics working group of the Italian Society of Human Genetics (SIGU), November 2011. Ultrasound Obstet Gynecol 2012;39:384-388.

4. Lee CN, Lin SY, Lin CH, Shih JC, Lin TH, Su YN. Clinical utility of array comparative genomic hybridisation for prenatal diagnosis: a cohort study of 3171 pregnancies. BJOG 2012;119:614-625.

5. Shaffer LG, Dabell MP, Rosenfeld JA, et al. Referral patterns for microarray testing in prenatal diagnosis. Prenat Diagn 2012;32:344-350.

6. Wapner RJ for the Prenatal Microarray NICHD Study Group. A multicenter, prospective, masked comparison of chromosomal microarray with standard karyotyping for routine and high risk prenatal diagnosis. Am J Obstet Gynecol 2012;206:S2.

7. Lippman A. Prenatal genetic testing and geneticization: mother matters for all. Fetal Diagn Ther 1993;8(suppl 1):175-188.

8. Sandelowski M, Barroso J. The travesty of choosing after positive prenatal diagnosis. J Obstet Gynecol Neonatal Nurs 2005;34:307-318.

9. Darilek S, Ward P, Pursley A, et al. Pre- and postnatal genetic testing by arraycomparative genomic hybridization: genetic counseling perspectives. Genet Med 2008;10:13-18.

10. Coppinger J, Alliman S, Lamb AN, Torchia BS, Bejjani BA, Shaffer LG. Whole-genome microarray analysis in prenatal specimens identifies clinically significant chromosome alterations without increase in results of unclear significance compared to targeted microarray. Prenat Diagn 2009;29:1156-1166.

11. Zuffardi $O$, Vetro A, Brady P, Vermeesch J. Array technology in prenatal diagnosis. Semin Fetal Neonatal Med 2011;16:94-98.

12. Lichtenbelt KD, Knoers NV, Schuring-Blom GH. From karyotyping to array-CGH in prenatal diagnosis. Cytogenet Genome Res 2011;135:241-250.

13. McGillivray G, Rosenfeld JA, McKinlay Gardner RJ, Gillam LH. Genetic counselling and ethical issues with chromosome microarray analysis in prenatal testing. Prenat Diagn 2012;32:389-395.

14. Kenen RH. The at-risk health status and technology: a diagnostic invitation and the 'gift' of knowing. Soc Sci Med 1996;42:1545-1553.

15. Marteau TM, Dormandy E. Facilitating informed choice in prenatal testing: how well are we doing? Am J Med Genet 2001;106:185-190.

16. Rapp R. Testing women, testing the fetus. New York: Routledge, 1999.

17. Sandelowski M, Jones LC. Healing fictions': stories of choosing in the aftermath of the detection of fetal anomalies. Soc Sci Med 1996;42:353-361.

18. Lalor J, Begley CM, Galavan E. Recasting Hope: a process of adaptation following fetal anomaly diagnosis. Soc Sci Med 2009;68:462-472.

19. Statham H, Solomou W, Chitty L. Prenatal diagnosis of fetal abnormality: psychological effects on women in low-risk pregnancies. Baillieres Best Pract Res Clin Obstet Gynaecol 2000;14:731-747.

20. Howard ED. Family-centered care in the context of fetal abnormality. J Perinat Neonatal Nurs 2006;20:237-242.

21. Sommerseth $E$, Sundby J. Women's experiences when ultrasound examinations give unexpected findings in the second trimester. Women Birth 2010;23: $111-116$.

22. Van den Veyver IB, Patel A, Shaw CA, et al. Clinical use of array comparative genomic hybridization $(\mathrm{aCGH})$ for prenatal diagnosis in 300 cases. Prenat Diagn 2009;29:29-39.

23. Fiorentino F, Caiazzo F, Napolitano S, et al. Introducing array comparative genomic hybridization into routine prenatal diagnosis practice: a prospective study on over 1000 consecutive clinical cases. Prenat Diagn 2011;31:1270-1282.

24. Hickerton $C L$, Aitken M, Hodgson J, Delatycki MB. "Did you find that out in time?": new life trajectories of parents who choose to continue a pregnancy where a genetic disorder is diagnosed or likely. Am J Med Genet $A$ 2012;158A:373-383.

25. Redlinger-Grosse K, Bernhardt BA, Berg K, Muenke M, Biesecker BB. The decision to continue: the experiences and needs of parents who receive a prenatal diagnosis of holoprosencephaly. Am J Med Genet 2002;112:369-378.

26. Tluczek A, Chevalier McKechnie A, Lynam PA. When the cystic fibrosis label does not fit: a modified uncertainty theory. Qual Health Res 2010; 20:209-223. 
27. DeLuca JM, Kearney MH, Norton SA, Arnold GL. Parents' experiences of expanded newborn screening evaluations. Pediatrics 2011;128:53-61.

28. Reiff $M$, Bernhardt BA, Mulchandani S, et al. "What does it mean?": uncertainties in understanding results of chromosomal microarray testing. Genet Med 2012:14:250-258.

29. Kleinveld JH, Ten Kate LP, van den Berg M, van Vugt JM, Timmermans DR. Does informed decision making influence psychological outcomes after receiving a positive screening outcome? Prenat Diagn 2009;29:271-273.

30. Farrell RM, Dolgin N, Flocke SA, Winbush V, Mercer MB, Simon C. Risk and uncertainty: shifting decision making for aneuploidy screening to the first trimester of pregnancy. Genet Med 2011;13:429-436.

31. Hunt $L M$, de Voogd KB, Castañeda $H$. The routine and the traumatic in prenatal genetic diagnosis: does clinical information inform patient decision-making? Patient Educ Couns 2005;56:302-312.

32. Fisher J. Supporting patients after disclosure of abnormal first trimester screening results. Curr Opin Obstet Gynecol 2012;24:109-113.

33. Farrelly E, Cho MK, Erby L, Roter D, Stenzel A, Ormond K. Genetic Counseling for Prenatal Testing: Where is the Discussion About Disability? I Genet Couns 2012; e-pub ahead of print 2 February 2012.
34. Parens $E$, Asch A. Disability rights critique of prenatal genetic testing: reflections and recommendations. Ment Retard Dev Disabil Res Rev 2003;9:40-47.

35. Bernhardt BA, Rushton CH, Carrese J, Pyeritz RE, Kolodner K, Geller $\mathrm{G}$. Distress and burnout among genetic service providers. Genet Med 2009;11:527-535.

36. Bernhardt BA, Silver R, Rushton CH, Micco E, Geller G. What keeps you up at night? Genetics professionals' distressing experiences in patient care. Genet Med 2010;12:289-297.

37. Riggs ER, Jackson L, Miller DT, Van Vooren S. Phenotypic information in genomic variant databases enhances clinical care and research: the International Standards for Cytogenomic Arrays Consortium experience. Hum Mutat 2012;33:787-796.

38. Savage MS, Mourad MJ, Wapner RJ. Evolving applications of microarray analysis in prenatal diagnosis. Curr Opin Obstet Gynecol 2011;23:103108.

39. Wapner RJ, Driscoll DA, Simpson JL. Integration of microarray technology into prenatal diagnosis: counselling issues generated during the NICHD clinical trial. Prenat Diagn 2012;32:396-400. 